
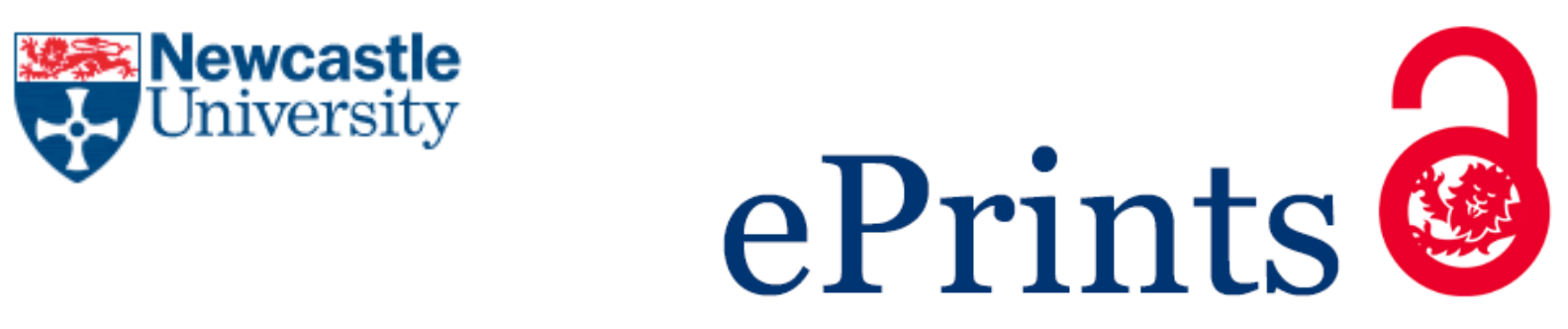

Liang Q, Hou J, Xia X. (2015)

Contradiction between the C-property and mass conservation in adaptive grid based shallow flow models: cause and solution. International Journal for Numerical Methods in Fluids DOI: $10.1002 / f l d .4005$

\title{
Copyright:
}

This is the accepted version of the above article which has been published in final form at: http://dx.doi.org/10.1002/fld.4005

Date deposited:

$25 / 02 / 2015$

Embargo release date:

23 February 2016

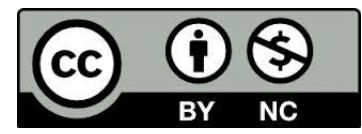

This work is licensed under a Creative Commons Attribution-NonCommercial 3.0 Unported License 


\section{Contradiction between Conservation Property and Mass Conservation in Adaptive Grid Based Shallow Flow Models: Cause and Solution}

\begin{tabular}{|r|l|}
\hline Journal: & International Journal for Numerical Methods in Fluids \\
\hline Manuscript ID: & Draft \\
\hline Wiley - Manuscript type: & Research Article \\
\hline Date Submitted by the Author: & $\mathrm{n}$ /a \\
\hline Complete List of Authors: & $\begin{array}{l}\text { Liang, Qiuhua; Hohai University, State Key Laboratory of Hydrology-Water } \\
\text { Resources and Hydraulic Engineering, } \text { University of Newcastle upon Tyne, } \\
\text { School of Civil Engineering \& Geosciences } \\
\text { Hou, Jingming; Newcastle Univeristy, School of civil engineering and } \\
\text { geosciences } \\
\text { Xia, Xilin; Newcastle Univeristy, School of Civil Engineering and } \\
\text { Geosciences }\end{array}$ \\
\hline Keywords: & $\begin{array}{l}\text { mass conservation, C-property, adaptive grids, shallow water flow models, } \\
\text { Finite volume, Free surface }\end{array}$ \\
\hline &
\end{tabular}




\title{
Contradiction between Conservation Property and Mass Conservation in Adaptive Grid Based Shallow Flow Models: Cause and Solution
}

\author{
Qiuhua Liang 1,2, Jingming Hou 2 *, Xilin Xia 2 \\ 1. State Key Laboratory of Hydrology-Water Resources and Hydraulic Engineering, Hohai University, Nanjing, China. \\ 2. School of Civil Engineering \& Geosciences, Newcastle University, Newcastle upon Tyne, NE1 7RU, UK
}

\begin{abstract}
SUMMARY
When performing shallow flow simulations on adaptive grids, the conservation property (C-property) and the mass conservation may not be simultaneously preserved, i.e. either C-property or mass conservation is likely to be violated following grid refining or coarsening. The cause of such a contradiction is analysed in detail in this work, which essentially links to the reconstruction of bed and flow information in those newly created cells during grid adaptation. An effective approach is subsequently proposed to resolve the contradiction by locally modifying the bed elevation in certain problematic cells when reconstructing flow variables and bed elevation by means of linear interpolation as part of the grid adaptation procedure. Copyright $\odot 0000$ John Wiley \& Sons, Ltd.
\end{abstract}

Received ...

KEY WORDS: mass conservation; C-property; adaptive grids; shallow water flow models

\section{INTRODUCTION}

Adaptive mesh refinement (AMR) has been broadly used to achieve high-resolution simulations only in those regions of interest, for example in [1, 2, 3, 4], in order to achieve a satisfactory level of numerical accuracy without compromising the overall computational efficiency. AMR may be commonly classified as block adaption and hierarchical grid adaption [5, 4]. These types of adaptive grids have been widely applied to solve the shallow water equations (SWE) for different applications, for example in [6, 7, 8, 9, 3]. In addition, [4] developed a new adaptive grid system that involves simple allocation of subdivision levels on given coarse background cells, removing the necessity of any complex data structure to store grid information.

Despite the improved computational efficiency compared with the uniformly refined mesh, an adaptive grid based SWE model may not simultaneously preserve the water surface conservation and the mass conservation during a simulation, as reported in $[9,5]$. The preservation of water surface is closely related to the conservation property (i.e. C-property) [10] of a SWE model. Cproperty essentially requires a model to numerically preserve a quiescent steady flow and is usually employed to reflect the well-balancedness of a numerical scheme, e.g. in $[11,12,13,14,15,16,17$, $18,19,20,21,22,23,24,25,26,27]$. The well-balancedness refers to the ability of a numerical scheme solving the SWE to balance the flux gradients and the source terms [28, 29, 30, 31]. In other words, the C-property affects the accuracy and numerical stability of a SWE model and thereby is

${ }^{*}$ Correspondence to: J. HOU, School of Civil Engineering \& Geosciences, Newcastle University, Newcastle upon Tyne, NE1 7RU, UK.

E-mail: jingming.hou@ncl.ac.uk

Copyright (C) 0000 John Wiley \& Sons, Ltd.

Prepared using fldauth.cls [Version: 2010/05/13 v2.00] 
an important feature such a model should possess. On the other hand, mass conservation is another key criterion reflecting the accuracy and reliability of numerical solutions and should be reinforced during a simulation. However, as previously mentioned, they may not coincide during a simulation involving AMR.

The contradiction between the C-property and mass conservation means that only one of them will be satisfied during AMR simulations, which may lead to numerical instability or loss of solution accuracy. For instance, considering a flow at rest over an uneven bed profile is simulated by an adaptive grid based SWE model that involves generation of refined mesh at wet-dry fronts to precisely capture the shoreline (Figure 1). While the mass conservation is reinforced on the newly refined cells, spurious waves are likely to be generated at the wet-dry fronts and propagate towards the wet domain, as demonstrated in Figure 2 where the simulation is done by the SWE model presented in [32] on the grid generated through applying the adaptive grid system in [4]. The generation of the unphysical waves will inevitably affect the accuracy of the simulation results.

Therefore, simultaneous preservation of mass conservation and C-property remains to be a research issue yet to be resolved. This work therefore aims to investigate more in-depth the cause of the contradiction between the C-property and the mass conservation during AMR simulations and subsequently propose an effective way to resolve the problem. The rest of the paper is arranged as follows: the cause of problem is analysed in detail in Section 2; a solution to the issue is proposed in Section 3; then Section 4 provides the mathematic proof and numerical test is carried out in Section 5; finally brief conclusions are drawn in Section 6 .

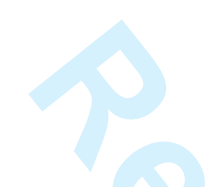

\section{THE CAUSE OF THE ISSUE}

In the context of SWE models, the well-balanced condition generally refers to the ability of a numerical scheme to properly balance the flux gradients and source terms $[28,29,30,31]$. In practice, a scheme may be considered to be well-balanced if it satisfies the conservation property (Cproperty) as defined in [10], i.e. when the quiescent steady state is preserved, at the computational level $[11,12,13,14,15,16,17,18,19,20,21,22,23,24,25,26,27]$. The surface water level must maintain to be constant when the flow velocity is zero in a quiescent flow. If a numerical scheme fails to satisfy the C-property, spurious momentum fluxes will be created and subsequently lead to inaccurate results or numerical instability.

On static grids, the C-property can be preserved by carefully designing techniques to balance the slope source terms and flux terms. These techniques include upwind discretization of the bed slope terms [33, 34], surface gradient methods (SGM) [35], flux correction method [36], hydrostatic reconstruction [37], mathematical balancing [18], slope flux method of [27], among others. However, the C-property is likely to be violated on dynamically adaptive grids when the mass conservation is also required, even when a proper balancing technique is implemented to deal with slope source terms $[9,5]$. This can be clearly demonstrated by the numerical results obtained for a flow at rest test as illustrated in Figure 2 (b). On the other hand, if the C-property is maintained during a simulation involving adaptive grids, the mass conservation is subjected to be violated.

In this work, water surface level, water depth and bed elevation, denoted respectively by $\eta, h$ and $z_{b}$, are used in the model and defined in Figure 3. The aforementioned issue of contradiction is likely to rise during the mesh refining or coarsening procedure that involves specifying flow variables and bed elevation in the new cells. Figure 4 (a) and (b) respectively plots the coarse parent cells and their refined sub-cells with relatively high water surface level, i.e. the water surface levels in the refined cells are higher than the bed elevations. In this case, as long as the water surface level and bed elevation are both linearly reconstructed in the refined cells and parent cell, the C-property and the mass conservation can be simultaneously satisfied. It should be noted that the linear reconstruction herein for bed elevation may be in the different forms according to the data availability. For example in Figure 4, if fine data are available, the value at a coarsened cell is averaged from all sub-cells 


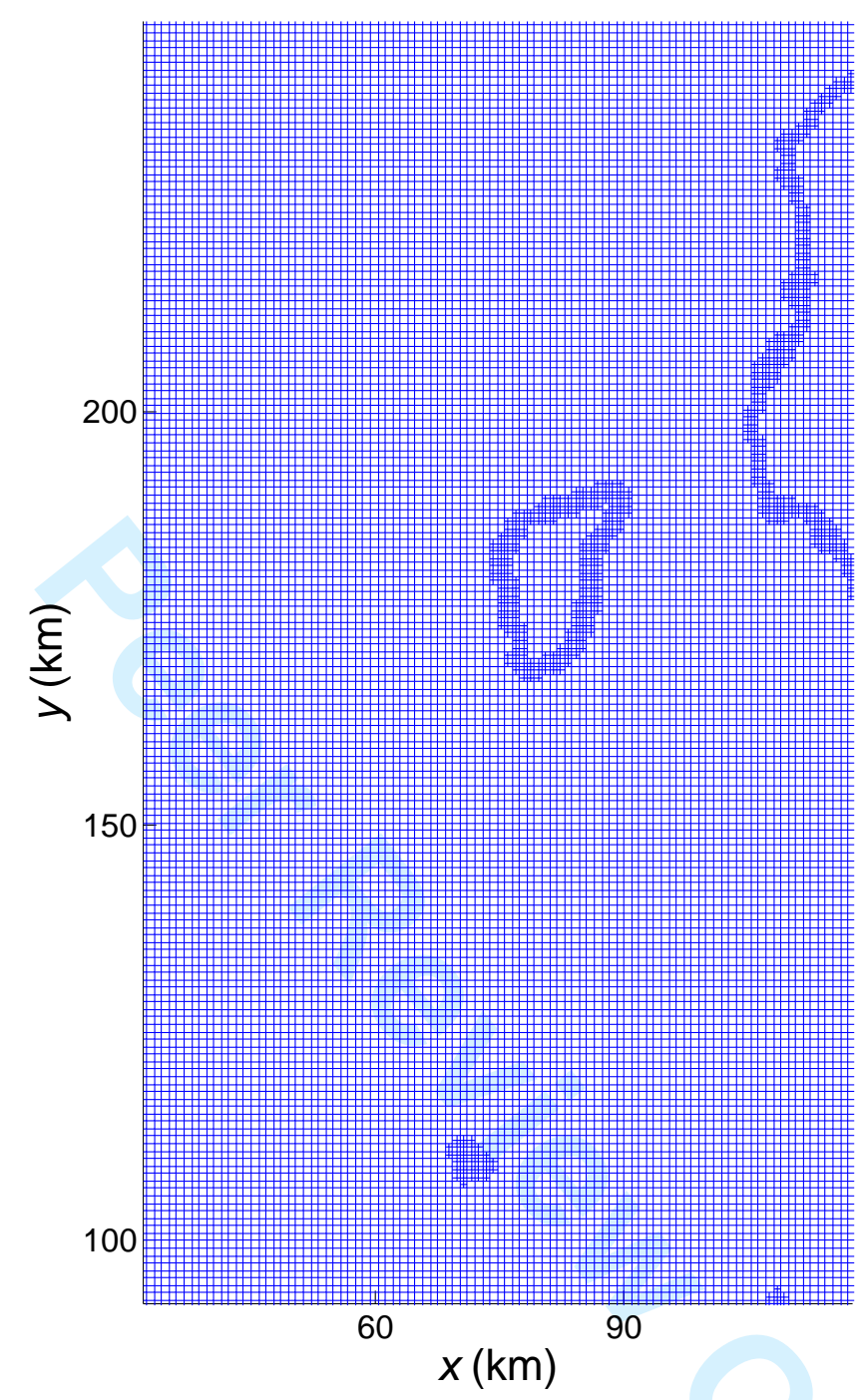

Figure 1. Refined meshes near to wet-dry fronts for the simulation as shown in Figure 2.

in grid coarsening, while the values in refined cells are directly determined from the available fine data in grid refining. Otherwise, if we only have coarse data, the values at refined cells are linearly interpolated from the available information in neighboring coarse cells, while the values at coarsened cells are the right coarse data in grid refining. The feature of preserving simultaneously the C-property and the mass conservation on the adaptive grids is proven in the cell $i$ as shown in Figure 4. The quiescent flow has a constant water surface level and so the water surface levels in the sub-cells $i_{-} 1$ and $i_{-} 2$ are the same as that in the corresponding coarse cell $i$, i.e.

$$
\eta_{i}=\eta_{i_{-} 1}=\eta_{i_{-} 2} .
$$

According to the linear relation, the bed elevation can be expressed by

$$
z_{b i}=\frac{1}{2}\left(z_{b i \_1}+z_{b i \_2}\right) .
$$

Assuming a 1D problem, the volume of water in the parent cell $i$ is calculated by

$$
m_{i}=h_{i} \Delta x
$$




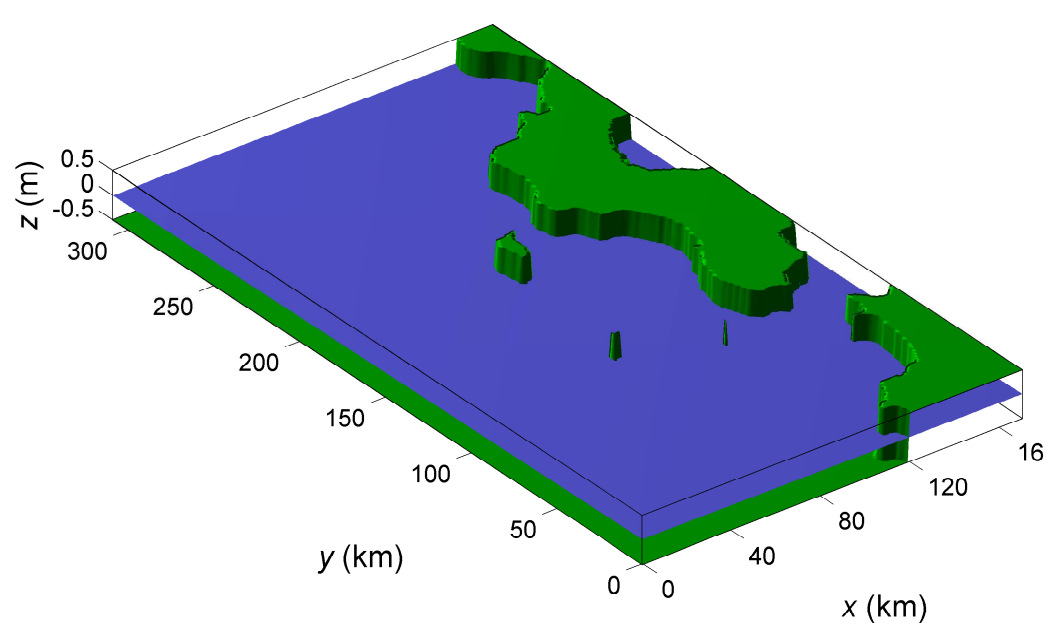

(a)

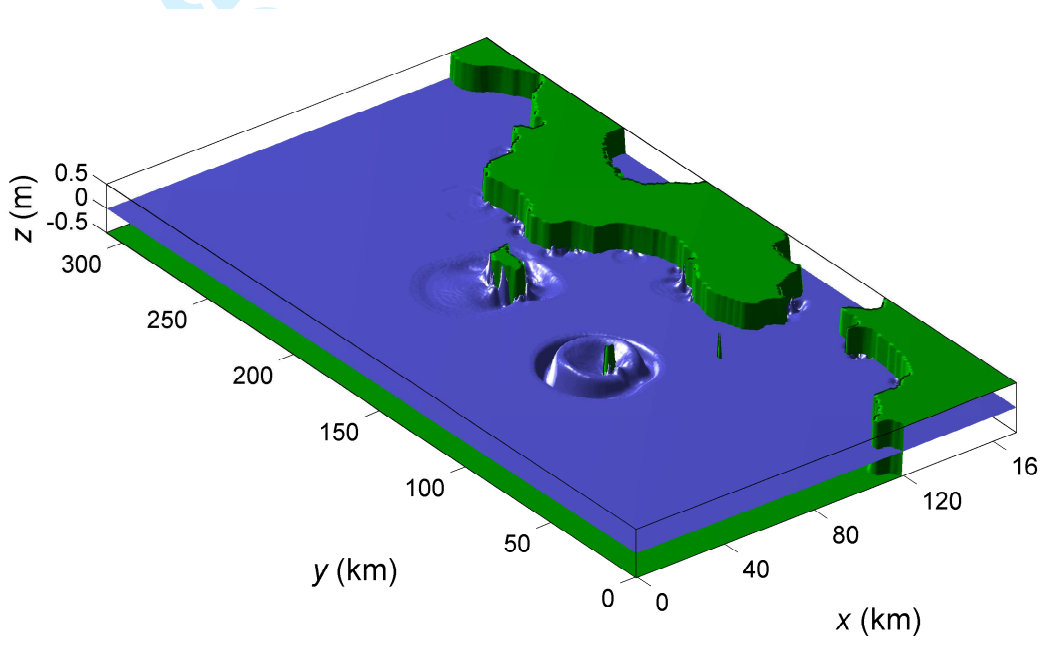

(b)

Figure 2. Predicted surface water level for flow at rest test at $t=200 \mathrm{~s}$ using: (a) uniform gird, (b) adaptive grid refined near to wet-dry fronts (Figure 1).

where $\Delta x$ represents the cell size. The total volume of water in the two sub-cells $i \_1$ and $i \_2$ is

$$
m_{i \_1}+m_{i_{-} 2}=h_{i_{-} 1} \frac{\Delta x}{2}+h_{i_{-} 2} \frac{\Delta x}{2} .
$$

From relations in equations (1) and (2) and $\eta=h+z_{b}$, we have $h_{i}=0.5\left(h_{i_{-} 1}+h_{i_{-} 2}\right)$ and in turn

$$
m_{i}=m_{i_{-} 1}+m_{i_{-} 2} .
$$

Equation (5) indicates that the mass conservation is satisfied; meanwhile the water surface level is preserved to ensure the C-property during grid adaption. However, this is not always the case as the reconstructed water surface level in a sub-cell may be lower than the bed elevation, for example the refined cell $i \_2$ in Figure 5 (b). Such a case may occur near wet-dry interface over uneven bed where the gradient of water depth is likely to be lower than that of bed elevation. Subsequently, if the water surface level is maintained in all cells to preserve the C-property, the reconstructed bed elevation in a sub-cell may rise above the water surface level, for instance, the bed level as shown 


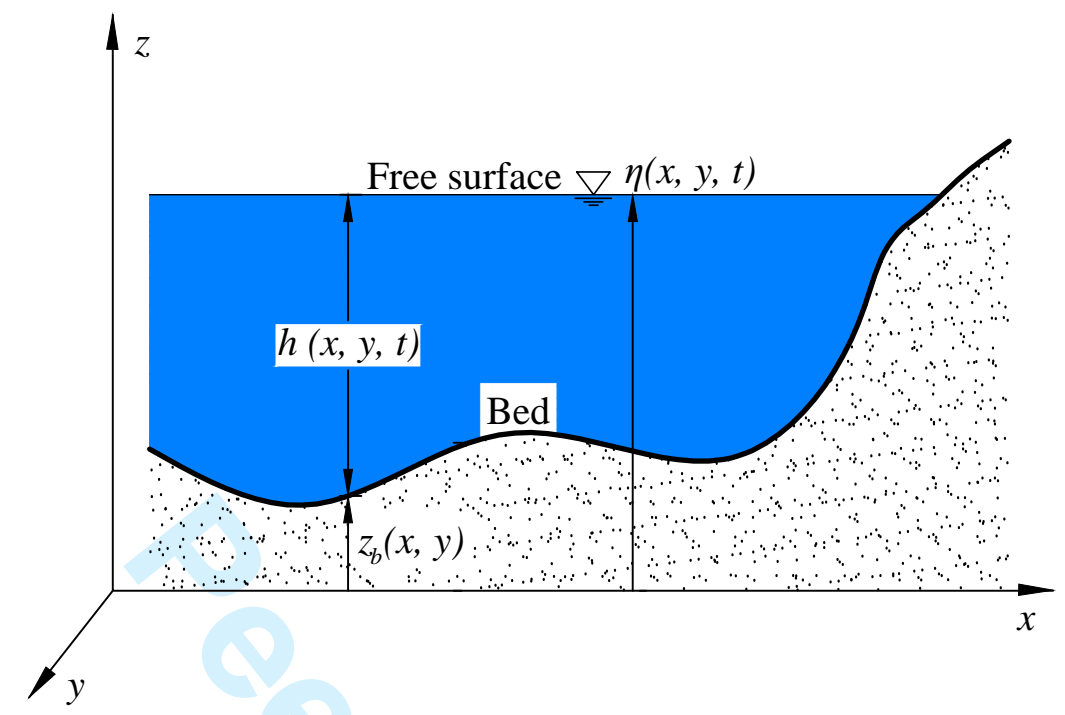

Figure 3. Notations of bed and flow variables for shallow water flows.

in cell $i_{-} 2$ in Figure 5 (b). The water pattern is obviously different from the previous case as a new dry sub-cell emerges. The total water volume in the two sub-cells is evaluated by

$$
m_{i_{-} 1}+m_{i_{-} 2}=h_{i_{-} 1} \frac{\Delta x}{2} \text {. }
$$

Since the volume of water in the parent cell $i$ is given by $m_{i}=h_{i} \Delta x$, the mass conservation is only preserved when $h_{i_{-} 1}=2 h_{i}$. As shown in Figure 5 (a) and (b), $h_{i}=\eta_{i}-z_{b i}$ and $h_{i_{-} 1}=\eta_{i_{-} 1}-z_{b i_{-} 1}$. If $h_{i_{-} 1}=2 h_{i}$ is assumed, we have

$$
\eta_{i_{-} 1}-z_{b i \_1}=2\left(\eta_{i}-z_{b i}\right) .
$$

As $\eta_{i_{-} 1}=\eta_{i}$, the above equation can be rearranged to become

$$
z_{b i}=\frac{1}{2}\left(\eta_{i}+z_{b i \_1}\right)
$$

According to equations (8) and (2), $\eta_{i}=z_{b i \_}$is derived. This is apparently incongruous to the fact that the reconstructed bed elevation is higher than the corresponding water surface level in subcell $i_{-} 2$. Therefore, the assumption of $h_{i_{-} 1}=2 h_{i}$ is invalid and the mass conservation is therefore violated. In contrast, if the mass conservation is preserved, the water surface level will no longer keep uniform as shown in Figure 5 (c), because the volume of water in sub-cell $i_{-} 1$ is equal to that in cell $i$ and thus can not guarantee the same water surface level as that in other wet cells. The contradiction can also be found in the grid coarsening procedure and the cause is similar. The above analysis clearly demonstrates the reason of why the C-property and the mass conservation cannot be satisfied at the same time on adaptive grids when the bed elevation in the sub-cells is lower than the reconstructed water surface level.

\section{PRESERVATION OF C-PROPERTY AND MASS CONSERVATION ON ADAPTIVE GRIDS}

It is known from last Section that the contradiction between the C-property and mass conservation is actually caused by coarsening or refining the cells with relatively small water depth over abrupt bed, e.g. the wet-dry fronts. As static grids will not give rise to this problem, a straightforward approach to handle the problem is to avoid grid adaption in the problematic regions. However, this approach 

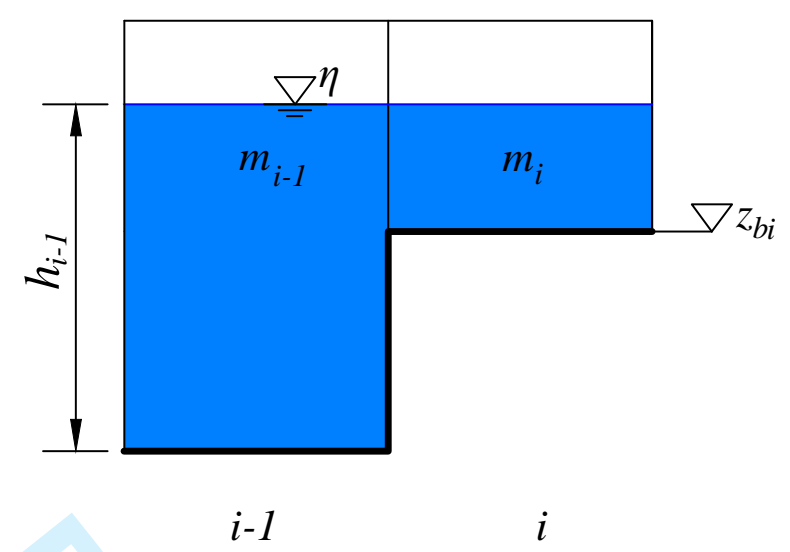

(a)

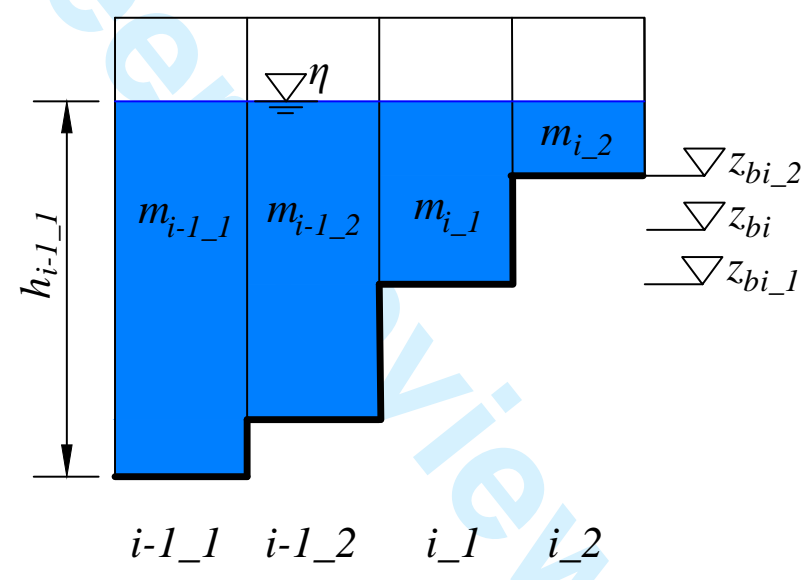

(b)

Figure 4. Reconstruction of flow information during grid refinement for case 1: (a) flow information in parent cells; (b) reconstructed flow information in sub-cells that satisfies the C-property and mass conservation.

of enforcing static grids inevitably compromises the merit of the adaptive grids that allow flexible change of grid resolution according to the needs. Herein, a novel approach is devised to resolve the contradiction between the $\mathrm{C}$-property and mass conservation on adaptive grids.

Last section reveals that the problem will not occur if the parent and sub-cells are all wet and the water depth/level and bed elevation are both reconstructed in a linear way which must be carried out in both refining and coarsening procedures. In engineering applications, however, the available coarse and refined bathymetric or topographic datasets may not have the linear relationship as required. For instance, if the given bed elevation in wet cell $i-1$ in Figure 4 is not equal to the averaged value of those in the two wet sub-cells $i-1 \_1$ and $i-1 \_2$, the bed elevation in the coarse wet cell should be modified to the averaged value from the corresponding fine cells, rather than use the available coarse ones, i.e.

$$
z_{b i-1}=\frac{1}{2}\left(z_{b i-1 \_1}+z_{b i-1 \_2}\right) .
$$

In the case of one or more sub-cells with reconstructed bed elevation higher than the corresponding water surface level, the contradiction may be resolved by modifying the bed elevation locally in the relevant cells during adaption. The modification for bed elevation is demonstrated herein in 1D cells in grid coarsening and refining to a higher and lower levels, respectively. 

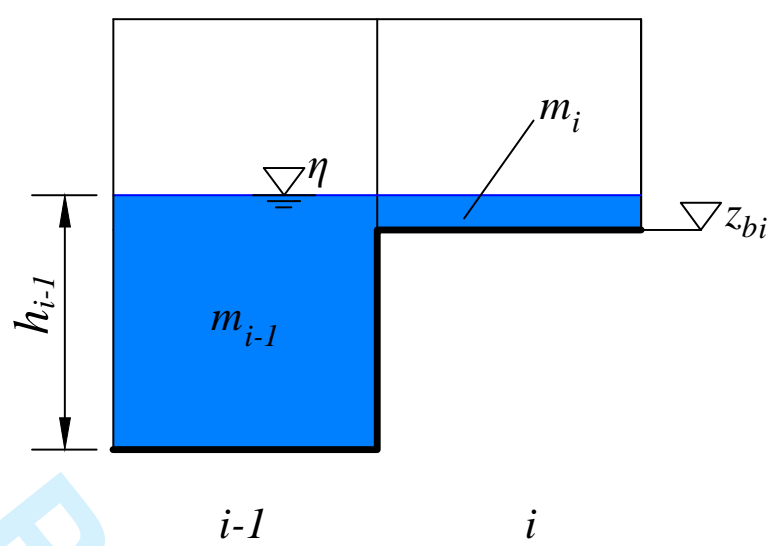

(a)

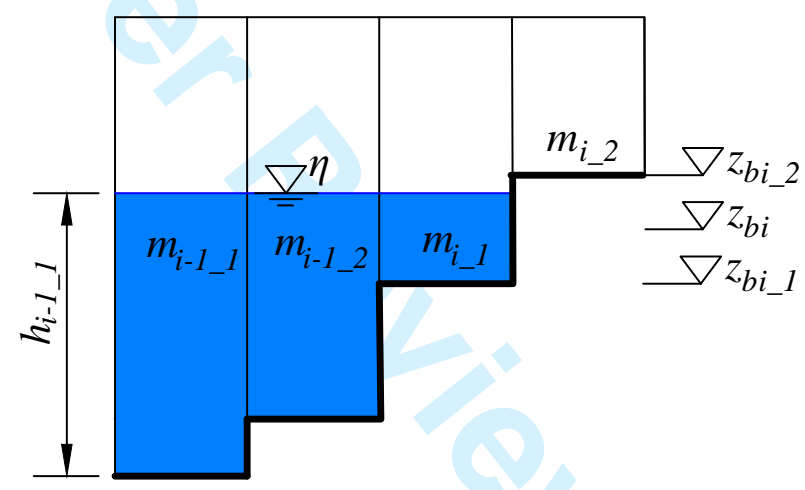

$$
\text { i-1_1 i-1_2 i_1 i_2 }
$$

(b)

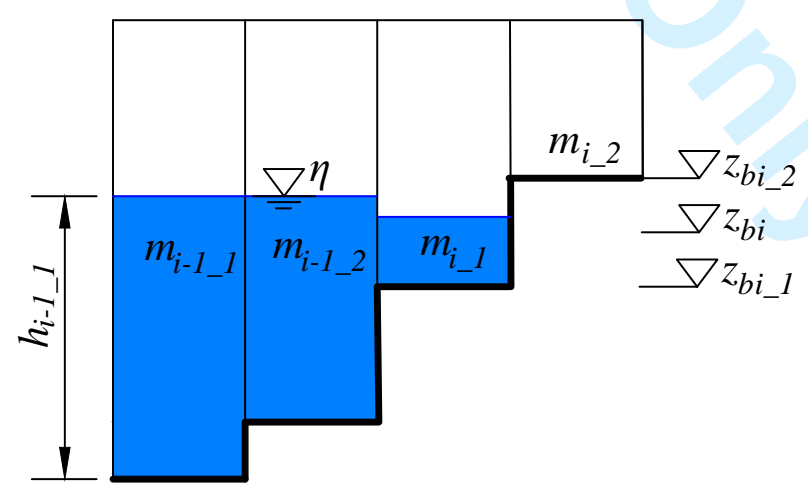

$$
\text { i-1_1 i-1_2 i_1 i_2 }
$$

(c)

Figure 5. Reconstruction of flow information during grid refinement for case 2: (a) flow information in parent cells; (b) (b) reconstructed flow information in sub-cells that satisfies the C-property, (c) reconstructed flow information in sub-cells that satisfies the mass conservation. 


\subsection{Local modification of bed elevation during cell refining}

Figure 6 shows how to modify the bed elevation in the two newly refined cells. When a cell is refined to a higher adaptation level, the flow variables and bed elevation must be reconstructed in the centres of two refined sub-cells, normally by means of linear construction. For example, the bed elevation and water surface level in sub-cell $i \_1$ can be respectively computed from

$$
\begin{gathered}
z_{b i \_1}=z_{b i}-\frac{\Delta x}{4} \nabla z_{b i}, \\
\eta_{i_{-} 1}=\eta_{i}-\frac{\Delta x}{4} \nabla \eta_{i},
\end{gathered}
$$

where $\Delta x$ is the cell dimension of the parent cell; $\nabla z_{b i}$ and $\nabla \eta_{i}$ denote the slope of the bed elevation and the water surface level, respectively, in the parent cell $i$. Other flow variables can be evaluated in the same way. $\nabla z_{b i}$ is termed as the original bed slop in Figure 6(b), which will be modified to a new bed slop $\bar{\nabla} z_{b i}$. In the same figure, the linearly reconstructed bed elevations of the two sub-cells are denoted as $z_{b i \_}$and $z_{b i_{-} 2}$. This case will be problematic as the reconstructed bed elevation is higher than the corresponding water surface level in sub-cell $i_{-} 2$ (Figure 6(b)). This will retain the C-property but violate the mass conservation as $h_{i_{-}} \neq 2 h_{i}$. In order to overcome the issue and ensure mass conservation, the bed elevation in the wet sub-cell $i_{-} 1$ may be locally modified by altering the bed slop $\nabla z_{b i}$ to be

$$
\bar{\nabla} z_{b i}=\frac{\min \left(z_{b i \_2}, \eta_{i \_2}\right)-z_{b i}}{0.25 \Delta x} .
$$

where the bed elevations and water surface level on the right hand side are obtained from linear reconstruction using the original slopes. Then the bed elevation in wet sub-cell $i_{-} 1$ is calculated based on $\bar{\nabla} z_{b i}$ as

$$
\bar{z}_{b i \_1}=z_{b i}-\frac{\Delta x}{4} \bar{\nabla} z_{b i} .
$$

For the dry sub-cell $i \_2$ whose bed elevation is higher than the water surface level, it is not necessary to modify its bed elevation as it neither affects the C-property nor mass conservation, as shown in Figure 6(c).

\subsection{Local modification of bed elevation during cell coarsening}

During grid coarsening, local bed modification must also be applied to resolve the contradiction between the C-property and mass conservation. Figure 7 illustrates the case when the two sub-cells $i \_1$ and $i \_2$ are coarsened to create a new (parent) cell $i$. If a dry sub-cell has a bed elevation higher than the linearly extrapolated water surface level, e.g. cell $i_{-} 2$ in Figure 7(b), simple linear reconstruction is likely to cause incorrect mass, i.e. $z_{b i}=0.5\left(z_{b i_{-} 1}+z_{b i_{-} 1}\right)$ leading to $h_{i} \neq 0.5 h_{i_{-} 1}$, as shown in Figure 7(b), although the uniform water surface level is preserved. Therefore it is necessary to modify the bed elevation in the coarsened cell $i$ to conserve mass by the following equation

$$
\bar{z}_{b i}=\frac{1}{2}\left[\min \left(z_{b i \_}, \eta_{i \_}\right)+\min \left(z_{b i \_}, \eta_{i \_1}\right)\right],
$$

The relationship between $\bar{z}_{b i}$ and $z_{b i}$ may be found in Figure 7(c).

\section{3. $2 D$ extension}

When refining a cell for 2D problems, the cell is normally divided into four sub-cells and we may use the available fine topographic data to accurately reflect the bed elevations in refined cells, rather than extrapolate them linearly from the coarse cell center like in equation (10). If this is the case, there may be no unique linear 2D slope for bed elevation in a coarse cell and thus we can not directly employ the approach proposed for 1D problem to modify the bed elevations for $2 \mathrm{D}$ problems. 


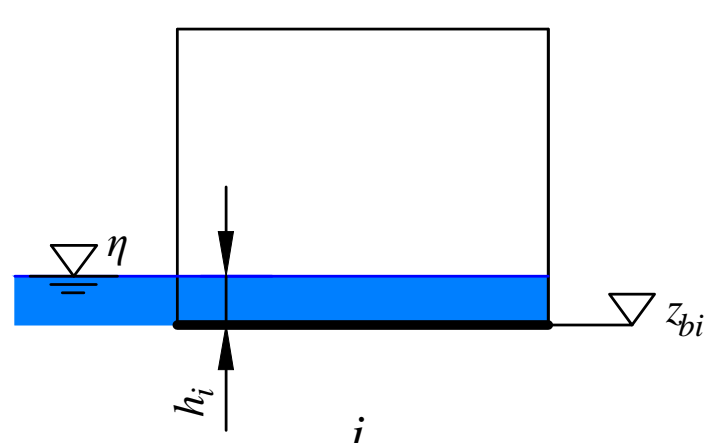

(a)

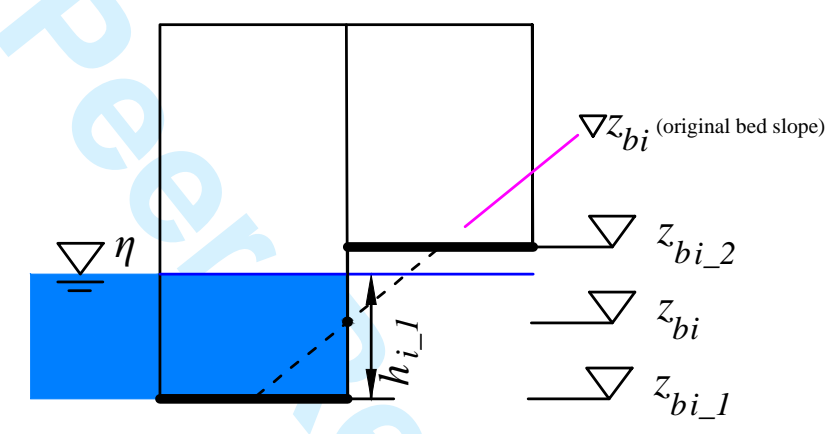

i_1 i_2

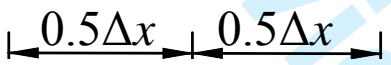

(b)
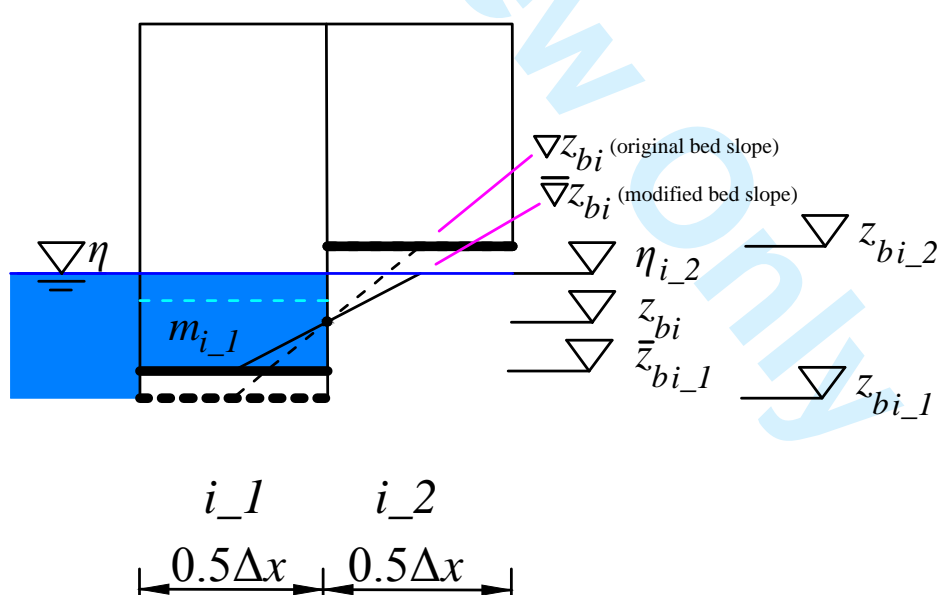

(c)

Figure 6. Local modification of bed elevation during cell refining: (a) original state of the flow in the parent cell; (b) linearly reconstructed flow information and bed elevation in the two sub-cells; (c) bed elevation after local bed modification.

To preserve the C-property and mass conservation for $2 \mathrm{D}$ problems through using the proposed approach, the bed elevation is modified in a 1D cross manner, e.g. the bed elevation in sub-cell $i \_1$ 


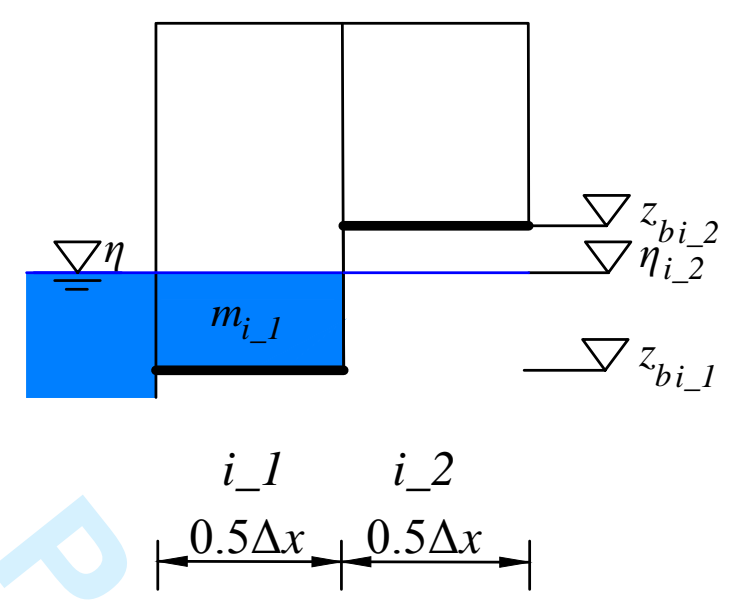

(a)

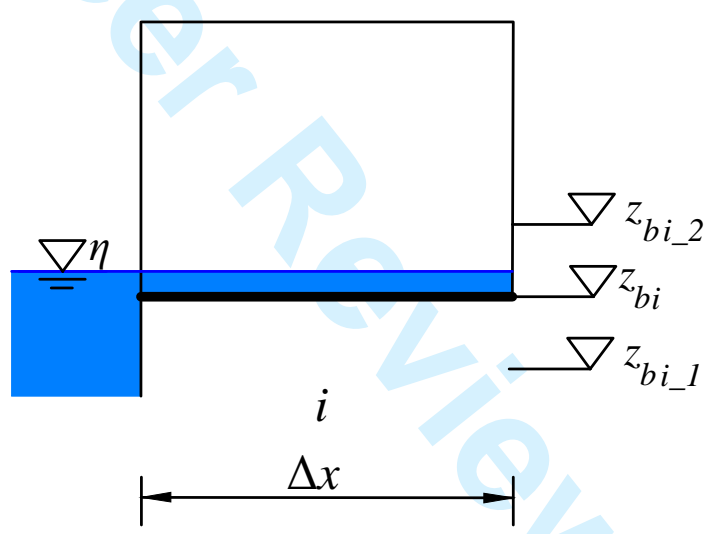

(b)

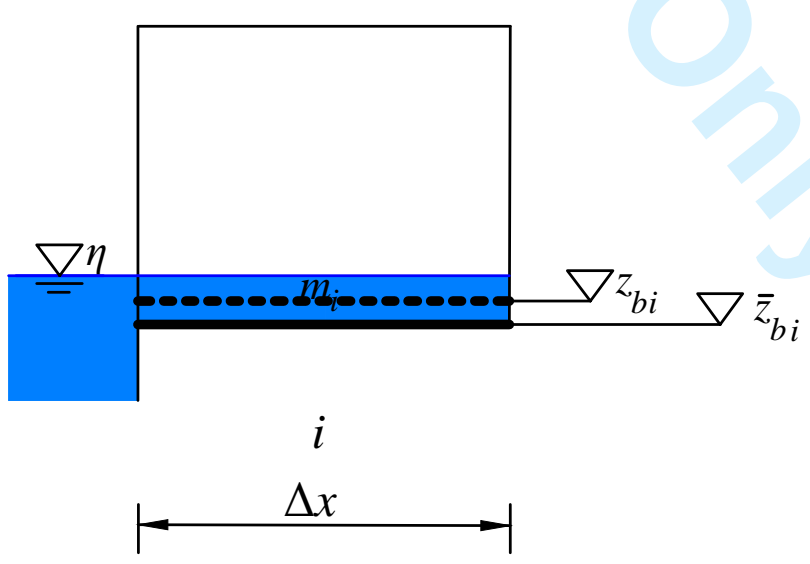

(c)

Figure 7. Local modification of bed elevation during cell coarsening: (a) original state of the flow in the two sub-cells; (b) linearly reconstructed flow information and bed elevation in the coarsened cell; (c) bed elevation after local bed modification. 


\section{PROOF OF THE C-PROPERTY AND THE MASS CONSERVATION}

The well-balanced cell-centered finite volume scheme presented in [32] is adopted in this work to solve the SWE. The C-property is preserved as long as the water surface level does not change after grid adaption for quiescent flow. As shown in Figures 6 and 7, the water surface levels in wet cells remain to be the same after grid adaption and consequently the C-property is satisfied when linear reconstruction is used to obtain the values of the bed elevation and flow variables in the newly created cells. The key point here is to prove whether the approach proposed in last section is capable of ensuring mass conservation.

When refining a cell, the volume of water in wet sub-cell $i_{-} 1$ can be evaluated by

$$
m_{i_{-} 1}=\frac{1}{2} h_{i_{-} 1} \Delta x=\frac{1}{2}\left(\eta_{i_{-1}}-\bar{z}_{b i_{-} 1}\right) \Delta x .
$$

From equations (12) and (13),

$$
\begin{aligned}
\bar{z}_{b i \_1} & =z_{b i}-\frac{\Delta x}{4} \bar{\nabla} z_{b i} \\
& =z_{b i}-\frac{\Delta x}{4}\left[\frac{4\left(\eta_{i_{-} 2}-z_{b i}\right)}{\Delta x}\right] \\
& =2 z_{b i}-\eta_{i_{-} 2} .
\end{aligned}
$$

Substituion of $\bar{z}_{b i \_}$into equation (16) gives 


$$
m_{i \_1}=\frac{1}{2}\left(\eta_{i \_1}+\eta_{i \_2}-2 z_{b i}\right) \Delta x .
$$

As $\nabla \eta=0.0, \eta_{i_{-} 1}$ and $\eta_{i_{-} 2}$ can be computed from equation (11) and subsequently $\eta_{i_{-} 1}=\eta_{i_{-} 2}=\eta_{i}$. Therefore, equation (16) can be rewritten as

$$
m_{i_{-} 1}=\frac{1}{2}\left(2 \eta_{i}-2 z_{b i}\right) \Delta x=h_{i} \Delta x .
$$

The water volume in cell $i$ is equal to $h_{i} \Delta x$ and that in the dry sub-cell $i_{-} 2$ is zero, i.e. $m_{i}=$ $m_{i_{-} 1}+m_{i_{-}}$, indicating that the new approach preserves mass conservation as well as C-property during grid refining.

As shown in Figure 7, the mass before coarsening the two sub-cells is expressed as

$$
m_{i \_1}+m_{i \_2}=h_{i \_1} \frac{\Delta x}{2}=\frac{1}{2}\left(\eta_{i_{-} 1}-z_{b i \_1}\right) \Delta x .
$$

After modifying locally the bed elevation using equation (7), the new mass in cell $i$ created following grid coarsening is

$$
m_{i}=h_{i} \Delta x=\left(\eta_{i}-\bar{z}_{b i}\right) \Delta x .
$$

From equation (14), we have $\bar{z}_{b i}=0.5\left(\eta_{i_{-} 1}+z_{b i_{-} 1}\right)$ and equation (21) is rearranged to be

$$
m_{i}=\frac{1}{2}\left(\eta_{i_{-} 1}-z_{b i \_1}\right) \Delta x .
$$

Therefore we have $m_{i}=m_{i_{-} 1}+m_{i_{-} 2}$, which proves that the new approach preserves mass conservation in grid coarsening.

\section{NUMERICAL TEST}

Through using the new approach in the quiescent flow simulation shown in Figure 2, the computed spurious waves on the adaptive grid completely disappear and no artificial motion is detected throughout the simulation, meanwhile, the total mass keeps constant during the simulation. To test the performance of the new approach which is designed to simultaneously preserve the C-property and mass conservation on adaptive grid in a dynamic case, the theoretical test developed in [38] is applied, as it describes a shallow water flow with wetting and drying over uneven bed. In this test case, the adaptive grid system proposed in [4] is under consideration but the adaption criterion is changed to use the water depth, i.e. the grid is refined if the water depth is smaller than a certain value $h_{c}$ and it is coarsened when the water depth is higher than $2 h_{c}\left(h_{c}=0.035 \mathrm{~m}\right.$ for this test case). The purpose of such a criterion is to undertake the adaption in the domain part involving wetting and drying which is likely to bring about the contradiction between the C-property and the mass conservation.

In this test case, the 2D frictionless parabolic bed topography is defined as

$$
z_{b}(x, y)=-h_{0}\left[1-\frac{\left(x-x_{0}\right)^{2}+\left(y-y_{0}\right)^{2}}{a^{2}}\right],
$$

where $\left[x_{0}, y_{0}\right]$ represents the center of the parabolic bowl; $h_{0}$ denotes the water depth at the domain center; $a$ is the distance from the center to the shoreline of zero elevation. The exact solution of this test case is given by

$$
\begin{aligned}
\eta(x, y, t) & =\frac{\sigma h_{0}}{a^{2}}\left[2\left(x-x_{0}\right) \cos (\omega t)\right. \\
& \left.+2\left(y-y_{0}\right) \sin (\omega t)-\sigma\right],
\end{aligned}
$$




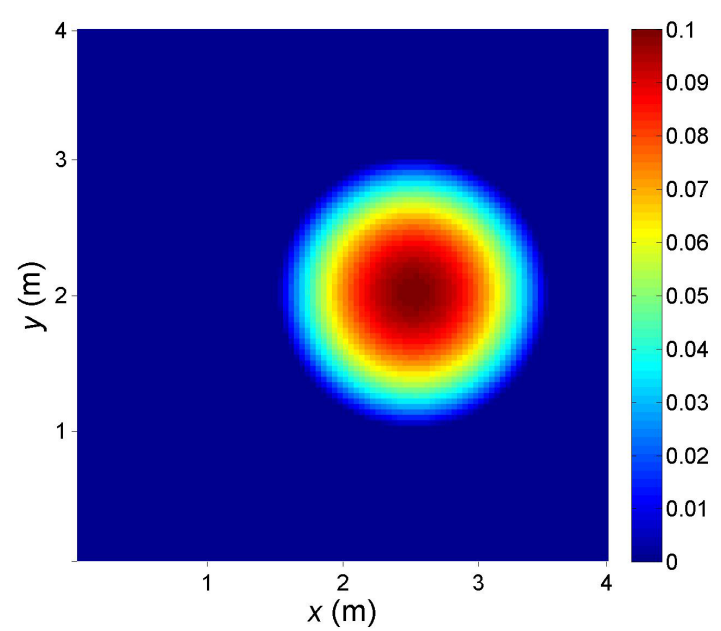

(a)

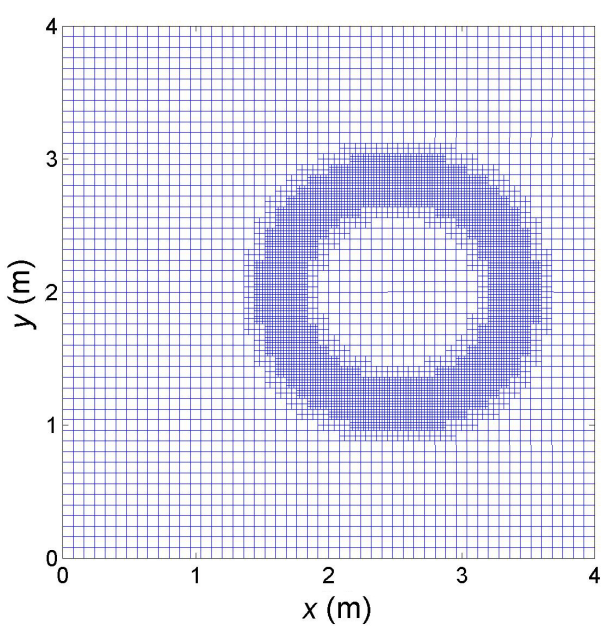

(b)

Figure 9. Computed water level (a) through using the new approach on the corresponding adaptive grid (b) at $t=4.49 \mathrm{~s}$.

$$
u(t)=-\omega \sigma \sin (\omega t), v(t)=\omega \sigma \cos (\omega t),
$$

in which $\sigma$ is a constant and $\omega=\sqrt{2 g h_{0}} / a$ is the frequency of the rotation.

In this work, the parameters are set to be $h_{0}=0.1 \mathrm{~m}, a=1.0 \mathrm{~m}$ and $\sigma=0.5 \mathrm{~m}$. A $4 \mathrm{~m} \times 4 \mathrm{~m}$ computational domain with the center of $(2 \mathrm{~m}, 2 \mathrm{~m})$ and four solid boundaries is chosen and the cell size varies between $0.08 \mathrm{~m}$ and $0.02 \mathrm{~m}$ according to the water depth as shown Figure 9. The SWE model presented in [32] is employed and the Courant number is 0.5 .

Figure 10 plots the computed water level by using the new approach in the cross section of $y=2.0 \mathrm{~m}$ at $t=10.17 \mathrm{~s}$ and $t=12.37 \mathrm{~s}$. A fairly good agreement with the exact solution is detected, indicating the new approach performs well in simulating wetting and drying over uneven bed on adaptive grid. If the conventional approach which only preserves the C-property is employed to compute the shallow water flow, less accurate result is produced comparing to the new approach, because the mass is not preserved in the grid adaption, see Figure 12. If the conventional approach preserving just the mass conservation in grid adaption, the result is even worsen as demonstrated in Figure 13, as the violation of the C-property is liable to cause spurious momentum which in turn leads to unrealistic flow pattern. In a word, the new approach is capable of handling such numerical problems and thus can improve the accuracy of shallow water flow models on adaptive grids.

\section{CONCLUSIONS}

This work intends to investigate the cause of contradiction between the C-property and mass conservation when numerically solving the SWEs on adaptive grids and to propose an effective approach to resolve the issue. It is found that the problem lies in the way by which the flow and bed information is reconstructed in the newly created cells. If all of refined sub-cells are wet, both the C-property and the mass conservation can be satisfied by using linear reconstruction. If the reconstructed water surface level is lower than the bed elevation in a sub-cell but higher than that of another sub-cell that is wet, the grid refining or coarsening will violate either the C-property or the mass conservation. Based on the detailed analysis of the cause of the problem, an effective approach is proposed to resolve this contradiction by means of local bed modification. The capability of the new approach in simultaneously preserving the $\mathrm{C}$-property and mass conservation on adaptive grids 


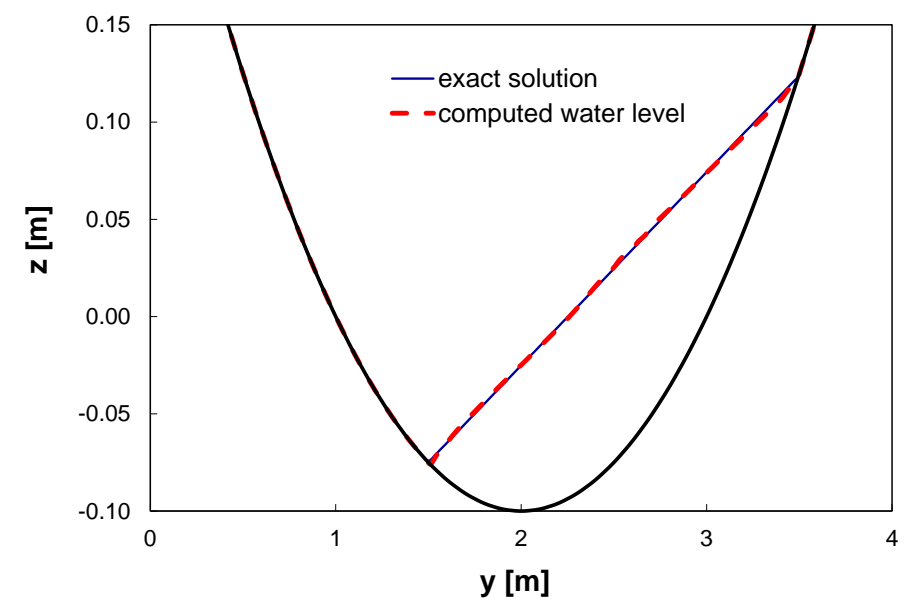

(a)

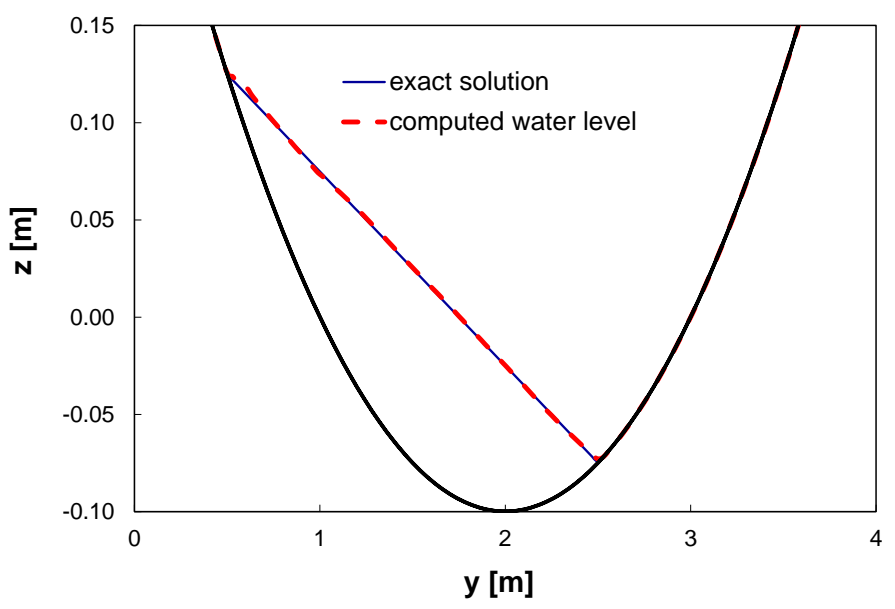

(b)

Figure 10. Computed water level through using the new approach in the cross section of $y=2.0 \mathrm{~m}$ at (a) $t=10.17 \mathrm{~s}$, (b) $t=12.37 \mathrm{~s}$.

is proven mathematically. Besides, a dynamic test case demonstrates that the new approach performs better in terms of accuracy than the conventional ones which preserve either the C-property or the mass conservation.

\section{ACKNOWLEDGEMENT}

The authors acknowledge the support from the Royal Society under the International Exchanges 2013 NSFC cost share scheme (IE131297). The first author is also supported by the National Natural Science Foundation of China through research grant (No. 51379074) and the Chinese Government through the 'Recruitment Program of Global Experts'. 


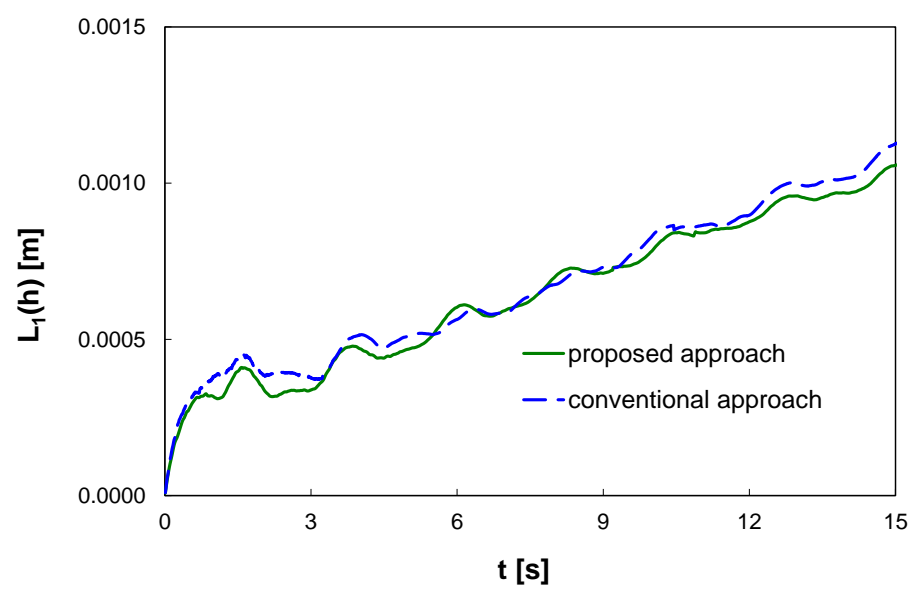

(a)

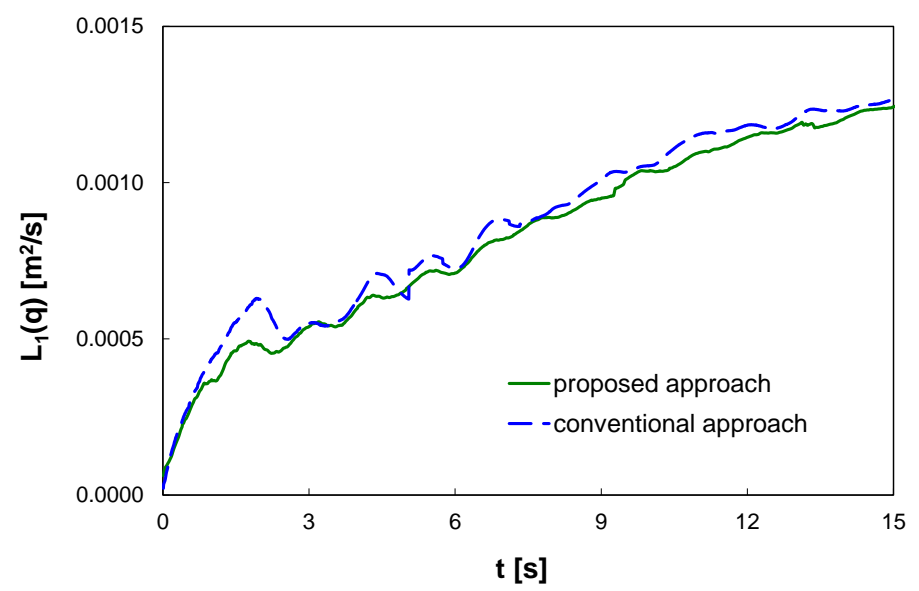

(b)

Figure 11. Computed $L_{1}$ errors through using the new approach and the conventional one only preserving the C-property: (a) $L_{1}(h)$, (b) $L_{1}(q)$ and $q=\sqrt{q_{x}^{2}+q_{y}^{2}}$.

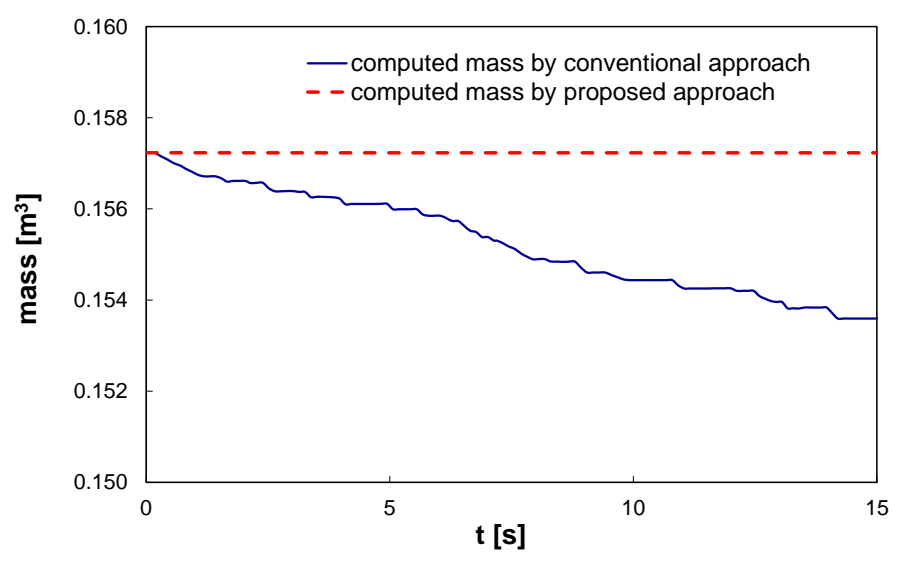

Figure 12. Computed total mass in terms of water volume through using the new approach and the conventional one only preserving the C-property. 


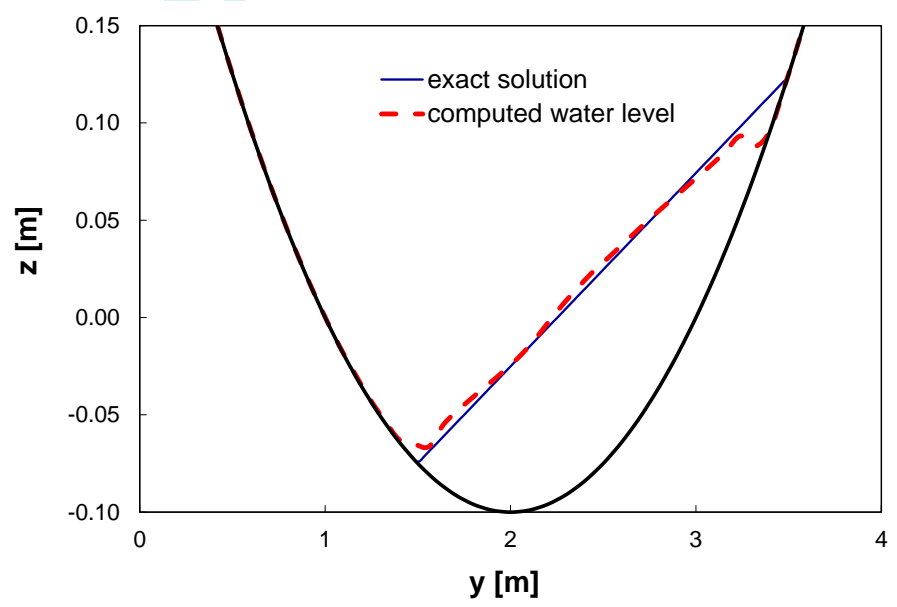

Figure 13. Computed water level through using the conventional one only preserving the mass conservation. 
1. Berger MJ, Oliger J. Adaptive mesh refinement for hyperbolic partial differential equations. Journal of Computational Physics Mar 1984; 53(3):484-512.

2. Yiu K, Greaves D, Cruz S, Saalehi A, Borthwick A. Quadtree grid generation: Information handling, boundary fitting and cfd applications. Computers \& Fluids Nov 1996; 25(8):759-769.

3. Lee WK, Borthwick AG, Taylor PH. A fast adaptive quadtree scheme for a two-layer shallow water model. Journal of Computational Physics Jun 2011; 230(12):4848-4870.

4. Liang Q. A simplified adaptive cartesian grid system for solving the $2 \mathrm{D}$ shallow water equations. International Journal for Numerical Methods in Fluids May 2012; 69(2):442-458.

5. Popinet S. Quadtree-adaptive tsunami modelling. Ocean Dynamics 2011; 61:1261-1285.

6. Rogers B, Fujihara M, Borthwick AGL. Adaptive q-tree godunov-type scheme for shallow water equations. International Journal for Numerical Methods in Fluids Feb 2001; 35(3):247-280.

7. Popinet S. Gerris: a tree-based adaptive solver for the incompressible Euler equations in complex geometries. Journal of Computational Physics Sep 2003; 190(2):572-600.

8. George DL, LeVeque RJ. High-Resolution Methods and Adaptive Refinement for Tsunami Propagation and Inundation, Hyperbolic Problems: Theory, Numerics, Applications. Springer-Verlag, 2008.

9. Liang Q, Borthwick AG. Adaptive quadtree simulation of shallow flows with wet dry fronts over complex topography. Computers \& Fluids 2009; 38:221-234.

10. Bermudez A, Vazquez ME. Upwind methods for hyperbolic conservation laws with source terms. Computers \& Fluids 1994; 23:1049-1071.

11. Audusse E, Bristeau MO. A well-balanced positivity preserving second-order scheme for shallow water flows on unstructured meshes. Journal of Computational Physics 2005; 206:311-333.

12. Ern A, Piperno S, Djadel K. A well-balanced runge-kutta discontinuous galerkin method for the shallow-water equations with flooding and drying. International Journal for Numerical Methods in Fluids 2008; 58:1-25.

13. Kim DH, Cho YS, Kim HJ. Well-balanced scheme between flux and source terms for computation of shallow-water equations over irregular bathymetry. Journal of Hydraulic Engineering 2008; 134:277-290.

14. Delis AI, Kazolea M, Kampanis NA. A robust high-resolution finite volume scheme for the simulation of long waves over complex domains. International Journal for Numerical Methods in Fluids 2008; 56:419-452.

15. Nikolos I, Delis A. An unstructured node-centered finite volume scheme for shallow water flows with wet/dry fronts over complex topography. Computer Methods in Applied Mechanics and Engineering 2009; 198:3723-3750.

16. Delis AI, Nikolos IK, Kazolea M. Performance and comparison of cell-centered and node-centered unstructured finite volume discretizations for shallow water free surface flows. Archives of Computational Methods in Engineering 2011; 18:57-118.

17. Delis AI, Nikolos IK. A novel multidimensional solution reconstruction and edge-based limiting procedure for unstructured cell-centered finite volumes with application to shallow water dynamics. International Journal for Numerical Methods in Fluids 2013; 71:584-633.

18. Liang Q, Marche F. Numerical resolution of well-balanced shallow water equations with complex source terms. Advances in Water Resources 2009; 32:873-884.

19. Song L, Zhou J, Li Q, Yang X, Zhang Y. An unstructured finite volume model for dam-break floods with wet/dry fronts over complex topography. International Journal for Numerical Methods in Fluids 2011; 67:960-980.

20. Song L, Zhou J, Guo J, Zou Q, Liu Y. A robust well-balanced finite volume model for shallownext term water previous termflowsnext term with wetting and drying over irregular terrain. Advances in Water Resources 2011; 34:915-932.

21. Benkhaldoun F, Elmahi I, Seaid M. Well-balanced finite volume schemes for pollutant transport by shallow water equations on unstructured meshes. Journal of Computational Physics 2007; 226:180-203.

22. Benkhaldoun F, Sahmim S, Seaid M. A two-dimensional finite volume morphodynamic model on unstructured triangular grids. International Journal for Numerical Methods in Fluids 2010; 63:1296-1327.

23. Xing Y, Shu CW. High-order finite volume weno schemes for the shallow water equations with dry states. Advances in Water Resources 2011; 34:1026-1038.

24. Canestrelli A, Fagherazzi S, Lanzoni S. A mass-conservative centered finite volume model for solving twodimensional two-layer shallow water equations for fluid mud propagation over varying topography and dry areas. Advances in Water Resources 2012; 40:54-70.

25. Pu JH, Cheng NS, Tan SK, Shao S. Source term treatment of SWes using surface gradient upwind method. Journal of Hydraulic Research 2012; 50:145-153.

26. Caleffi V. A new well-balanced Hermite weighted essentially non-oscillatory scheme for shallow water equations. International Journal for Numerical Methods in Fluids 2011; 67:1135-1159.

27. Hou J, Liang Q, Simons F, Hinkelmann R. A 2D well-balanced shallow flow model for unstructured grids with novel slope source term treatment. Advances in Water Resources 2013; 52:107-131.

28. Greenberg JM, Leroux AY. A well-balanced scheme for the numerical processing of source terms in hyperbolic equations. SIAM Journal on Numerical Analysis 1996; 33:1-16.

29. Vazquez-Cendon ME. Improved treatment of source terms in upwind schemes for the shallow water equations in channels with irregular geometry. Journal of Computational Physics 1999; 148:497-526.

30. Parés C, Castro M. On the well-balance property of Roe's method for nonconservative hyperbolic systems. Applications to shallow-water systems. ESAIM: Mathematical Modelling and Numerical Analysis 2004; 38:821852 .

31. Gallardo JM, Parés C, Castro M. On a well-balanced high-order finite volume scheme for shallow water equations with topography and dry areas. Journal of Computational Physics 2007; 227:574-601.

32. Liang Q. Flood simulation using a well-balanced shallow flow model. Journal of Hydraulic Engineering 2010; 136:669-675. 
33. Hubbard ME, Garcia-Navarro. Flux difference splitting and the balancing of source terms and flux gradients. Journal of Computational Physics 2000; 165:89-125.

34. Brufau P, Vázquez-Cendón ME, García-Navarro P. A numerical model for the flooding and drying of irregular domains. International Journal for Numerical Methods in Fluids 2002; 39:247-275.

35. Zhou JG, Causon DM, Mingham CG, Ingram DM. The surface gradient method for the treatment of source terms in the shallow-water equations. Journal of Computational Physics 2001; 168:1-25.

36. Begnudelli L, Sanders BF. Unstructured grid finite-volume algorithm for shallow-water flow and scalar transport with wetting and drying. Journal of Hydraulic Engineering 2006; 132:371-384.

37. Audusse E, Bouchut F, Bristeau MO, Klein R, Perthame B. A fast and stable well-balanced scheme with hydrostatic reconstruction for shallow water flows. SIAM Journal on Scientific Computing 2004; 25:2050-2065.

38. Thacker WC. Some exact solutions to the nonlinear shallow-water wave equations. Journal of Fluid Mechanics 1981; 107:499-508. 